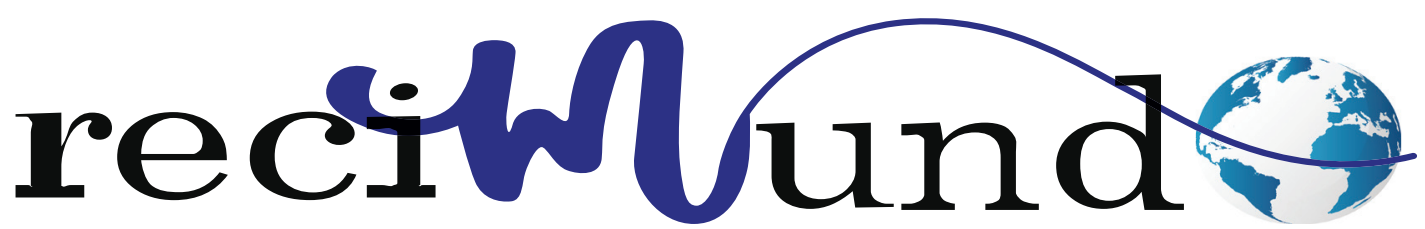

Revista Científica Mundo de la Investigación y el Conocimiento

DOI: 10.26820/recimundo/5.(2).abril.2021.203-211

URL: https://recimundo.com/index.php/es/article/view/1055

EDITORIAL: Saberes del Conocimiento

REVISTA: RECIMUNDO

ISSN: 2588-073X

TIPO DE INVESTIGACIÓN: Artículo de revisión

Código UNESCO: 32 Ciencias Médicas

PAGINAS: 203-211

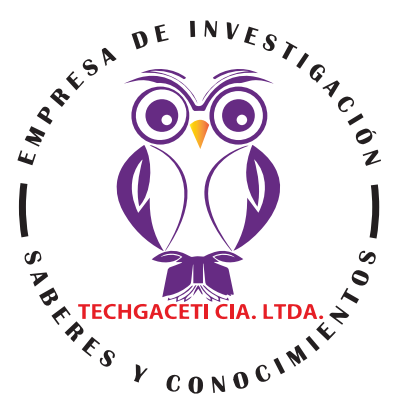

\title{
Ventilación mecánica en pacientes con COVID-19
}

Mechanical ventilation in patients with COVID-19

Ventilação mecânica em pacientes com COVID-19

Gloria Giomar Párraga Gusqui'; Cristina Alexandra Arias Muñoz²; Carlos Patricio Guamán Guaranga³;

Edison Antonio Rivera Moreira ${ }^{4}$

RECIBIDO: 15/01/2021 ACEPTADO: 20/03/2021 PUBLICADO: 01/04/2021

1. Médico de la Universidad Católica de Santiago de Guayaquil; Investigador Independiente; Guayaquil, Ecuador; gloria. parraga2326@gmail.com; (iD https://orcid.org/0000-0002-0263-9495

2. Médico de la Universidad de Guayaquil, Investigador Independiente; Guayaquil, Ecuador; cristinariasmunoz@gmail.com; (iD) https://orcid.org/0000-0001-7068-766X

3. Médico General de la Escuela Superior Politécnica de Chimborazo; Investigador Independiente; Riobamba, Ecuador; patog15031@hotmail.com; (iD https://orcid.org/0000-0002-9479-9161

4. Médico de la Universidad de Guayaquil, Investigador Independiente; Guayaquil, Ecuador; eddie_21_91@hotmail.com; (iD https://orcid.org/0000-0003-0579-7832

CORRESPONDENCIA

Gloria Giomar Párraga Gusqui

gloria.parraga2326@gmail.com

Guayaquil, Ecuador

() RECIMUNDO; Editorial Saberes del Conocimiento, 2021 


\section{RESUMEN}

El COVID-19 ha causado un revuelo a nivel mundial desde su aparición a finales del año 2019 de acuerdo a su rápida propagación por el mundo, convirtiéndose en pocos meses, en una pandemia que se ha mantenido hasta la actualidad. A pesar de los esfuerzos de la Organización Mundial de la Salud, todos los laboratorios y especialistas, el virus, sigue presente y en estudio debido a las nuevas variantes y mutaciones que se han registrado en los últimos meses. Por tal razón, el mundo está analizando cada cohorte y cada resultado de los pacientes con enfermedad por coronavirus (COVID-19), en particular los más gravemente enfermos que reciben ventilación mecánica. Los números que se han publicado están por todas partes y algunos de ellos, como la mortalidad muy alta, están causando pánico. Dos cuestiones importantes se encuentran en juego en estos estudios epidemiológicos. La primera es cuándo intubar y evaluar las tasas de intubación y ventilación mecánica para pacientes hospitalizados en cohortes de todo el mundo. El segundo es la mortalidad informada para los pacientes que reciben ventilación mecánica. La presentación e interpretación de los datos para ambos temas no es sencilla y nunca lo ha sido. Sin embargo, hay formas de mejorar la evaluación de estos estudios de cohortes.

Palabras clave: Ventilación mecánica, COVID-19, Insuficiencia respiratoria.

\section{ABSTRACT}

COVID-19 has caused a stir worldwide since its appearance at the end of 2019 according to its rapid spread throughout the world, becoming in a few months, a pandemic that has continued to this day. Despite the efforts of the World Health Organization, all laboratories and specialists, the virus is still present and under study due to the new variants and mutations that have been registered in recent months. For this reason, the world is analyzing each cohort and each outcome of patients with coronavirus disease (COVID-19), particularly the most seriously ill who receive mechanical ventilation. The numbers that have been published are all over the place and some of them, like the very high mortality, are causing panic. Two important issues are at stake in these epidemiological studies. The first is when to intubate and assess rates of intubation and mechanical ventilation for hospitalized patients in cohorts from around the world. The second is the reported mortality for patients receiving mechanical ventilation. The presentation and interpretation of the data for both subjects is not straightforward and never has been. However, there are ways to improve the evaluation of these cohort studies.

Keywords: Mechanical ventilation, COVID-19, Respiratory failure.

\section{RESUMO}

A COVID-19 tem causado uma agitação mundial desde seu surgimento no final de 2019, de acordo com sua rápida disseminação pelo mundo, tornando-se em poucos meses, uma pandemia que tem continuado até hoje. Apesar dos esforços da Organização Mundial da Saúde, de todos os laboratórios e especialistas, o vírus ainda está presente e em estudo devido às novas variantes e mutações que têm sido registradas nos últimos meses. Por esta razão, o mundo está analisando cada coorte e cada resultado de pacientes com doença coronavírus (COVID-19), particularmente os mais seriamente doentes que recebem ventilação mecânica. Os números que foram publicados estão por toda parte e alguns deles, como a mortalidade muito alta, estão causando pânico. Duas questões importantes estão em jogo nestes estudos epidemiológicos. A primeira é quando intubar e avaliar as taxas de entubação e ventilação mecânica para pacientes hospitalizados em coortes de todo o mundo. A segunda é a mortalidade relatada para os pacientes que recebem ventilação mecânica. A apresentação e interpretação dos dados para ambos os sujeitos não é simples e nunca foi. Entretanto, há maneiras de melhorar a avaliação destes estudos de coorte.

Palavras-chave: Ventilação mecânica, COVID-19, Falha respiratória. 


\section{Introducción}

La ventilación mecánica (VM) es fundamental para mantener la vida en casos de insuficiencia respiratoria grave. Los orígenes del MV se remontan al siglo XVI, cuando Vesalio describió la técnica en el libro De Humani Corporis Fabrica. "Los ventiladores de presión negativa se desarrollaron a finales del siglo XIX, mientras que la VM invasiva, como la conocemos, surgió en respuesta a la pandemia de poliomielitis de 1952 en Dinamarca" (Slutsky, 2015). En ese momento, el anestesiólogo Bjorn Ibsen utilizó la traqueotomía y la ventilación manual con presión positiva en pacientes con formas graves de la enfermedad y parálisis de los músculos respiratorios, lo que redujo la letalidad del $97 \%$ al $40 \%$.

A partir de entonces, la VM llegó a ser reconocida como una técnica que salva vidas, y su historia se confunde con la de las UCI. "Desde entonces ha evolucionado desde un soporte básicamente orientado a normalizar el intercambio gaseoso a una técnica capaz de hacerlo, sin dañar los pulmones, sin comprometer la fisiología del sistema cardiovascular o de otros órganos, y sin causar disfunción diafragmática" (Slutsky, 2015), asegurando así la resolución de la enfermedad de base, proporcionando una buena sincronía paciente-ventilador y reduciendo la necesidad de sedación.

Después de casi 70 años, la VM ahora enfrenta su mayor desafío: la nueva pandemia de la enfermedad por coronavirus 2019 (COVID-19). La poliomielitis se acompañó de acidosis respiratoria por insuficiencia neuromuscular, mientras que la neumonía por el nuevo coronavirus causa daño severo al parénquima pulmonar e hipoxemia severa, que a menudo es refractaria a las intervenciones habituales, en $10-20 \%$ de los casos. ( World Health Organization, 2020)

Algunos pocos meses después de que se reportó el primer caso de COVID-19 en Chi- na, la enfermedad fue declarada pandemia, y al 8 de junio de 2020 se habían reportado más de 6.9 millones de casos confirmados y 400.469 muertes ( World Health Organization, 2020). Para esa misma fecha, 645.771 casos confirmados y se habían reportado 35.026 defunciones en Brasil, que pasó a ocupar el tercer lugar en términos de número absoluto de defunciones a nivel mundial.

Estas cifras, que se habían alcanzado en tan poco tiempo, muestran la alta tasa de contagio del virus que causa COVID-19, denominado síndrome respiratorio agudo severo coronavirus 2 (SARS-CoV-2). Aunque las formas graves de la enfermedad se presentan en solo una pequeña fracción de los pacientes, el número absoluto de estos pacientes es considerable y podría eventualmente causar el colapso de los sistemas de atención médica. La limitación estructural más crítica es la escasez de camas de UCI y ventiladores mecánicos, siendo el soporte ventilatorio el meollo del problema.

Además, existen restricciones en el uso de ventilación no invasiva y cánulas nasales de alto flujo debido al riesgo de aerosolizar el virus en el ambiente e infectar a miembros del equipo multidisciplinario u otros pacientes. Los pacientes con COVID-19 pueden requerir VM durante dos a cuatro semanas. "Además, las complicaciones, como la neumonía asociada al respirador, la tromboembolia pulmonar, el delirio y la asincronía paciente-ventilador que es difícil de resolver, pueden aumentar la morbilidad y la mortalidad" (Berlin, Gulick, \& Martinez, 2020).

Los desafíos para proporcionar VM de manera segura incluyen mantener el suministro de materiales, como equipos de protección personal, accesorios de VM (como filtros y circuitos) y medicamentos (para sedación, analgesia y bloqueos neuromusculares), así como la necesidad de apoyo de los servicios de ingeniería clínica. 


\section{Metodología}

Para el desarrollo de este proceso investigativo, se plantea como metodología la encaminada hacia una orientación científica particular que se encuentra determinada por la necesidad de indagar en forma precisa y coherente una situación, en tal sentido Davila, (2015) define la metodología "como aquellos pasos anteriores que son seleccionados por el investigador para lograr resultados favorables que le ayuden a plantear nuevas ideas" (p.66)

Lo citado por el autor, lleva a entender que el desarrollo de la acción investigativa busca simplemente coordinar acciones enmarcadas en una revisión bibliográfica con el fin de complementar ideas previas relacionadas Ventilación mecánica en pacientes con COVID-19 a través de una revisión de literatura, para así finalmente elaborar un cuerpo de consideraciones generales que ayuden a ampliar el interés propuesto.

\section{Tipo de Investigación}

Dentro de toda práctica investigativa, se precisan acciones de carácter metodológico mediante las cuales se logra conocer y proyectar los eventos posibles que la determinan. En este sentido, la presente investigación corresponde al tipo documental, definido por Castro (2016), "se ocupa del estudio de problemas planteados a nivel teórico, la información requerida para abordarlos se encuentra básicamente en materiales impresos, audiovisuales y / o electrónicos". (p.41).

En consideración a esta definición, la orientación metodológica incluye la oportunidad de cumplir con una serie de actividades inherentes a la revisión y lectura de diversos documentos, donde se encuentran ideas explicitas relacionadas con los tópicos encargados de identificar una característica inmersa en el estudio. Por lo tanto, se realizaron continuas interpretaciones con el claro propósito de revisar aquellas apreciaciones propuestas por diferentes investigadores en relación al tema de interés, para luego dar la respectiva argumentación a los planteamientos, en función a las necesidades encontradas en la investigación, apoyados en las herramientas tecnológicas para la búsqueda de trabajos con valor científico disponibles en la web que tenían conexión con el objetivo principal de la investigación.

\section{Fuentes Documentales}

El análisis correspondiente a las características que predomina en el tema seleccionado, llevan a incluir diferentes fuentes documentales encargadas de darle el respectivo valor científico y en ese sentido cumplir con la valoración de los hechos a fin de generar nuevos criterios que sirven de referencia a otros procesos investigativos. Para Castro,(2016) las fuentes incorporadas en la investigación documental o bibliográfica, "representa la suma de materiales sistemáticos que son revisados en forma rigurosa y profunda para llegar a un análisis del fenómeno" (p.41). Por lo tanto, se procedió a cumplir con la lectura previa determinada para encontrar aquellos aspectos estrechamente vinculados con el tema, con el fin de explicar mediante un desarrollo las respectivas apreciaciones generales de importancia.

\section{Técnicas para la Recolección de la Infor- mación}

La conducción de la investigación para ser realizada en función a las particularidades que determinan a los estudios documentales, tiene como fin el desarrollo de un conjunto de acciones encargadas de llevar a la selección de técnicas estrechamente vinculadas con las características del estudio. Bolívar, (2015), refiere, que es "una técnica particular para aportar ayuda a los procedimientos de selección de las ideas primarias y secundarias". (p.71). 
Tal como lo expresa, Bolívar, (2015) "Las técnicas documentales proporcionan las herramientas esenciales y determinantes para responder a los objetivos formulados y llegar a resultados efectivos" (p. 58). Es decir, para responder con eficiencia a las necesidades investigativas, se introdujeron como técnica de recolección el método inductivo, que hizo posible llevar a cabo una valoración de los hechos de forma particular para llegar a la explicación desde una visión general. El autor Bolívar, (2015) tambien expresa que las técnicas de procesamiento de datos en los estudios documentales "son las encargadas de ofrecer al investigador la visión o pasos que deben cumplir durante su ejercicio, cada una de ellas debe estar en correspondencia con el nivel a emplear" (p. 123). Esto indica, que para llevar a cabo el procesamiento de los datos obtenidos una vez aplicadas las técnicas seleccionadas, tales como: fichas de resumen, textual, registros descriptivos entre otros, los mismos se deben ajustar al nivel que ha sido seleccionado.

\section{Resultados}

\section{La Decisión de Ventilar}

Los autores Gattinoni, Chiumello, Caironi, Busana, Romitti, \& Brazzi, (2020) afirmaron que "los estadounidenses habían puesto a sus pacientes en los respiradores demasiado pronto; ciertamente, no habrían sido ventilados en Copenhague. No es de extrañar que sobrevivieran, afirmó, porque en primer lugar no necesitaban tratamiento en tiempos de polio". El mismo debate que se tiene ahora "con respecto al uso temprano versus tardío de la ventilación mecánica y cuándo los pacientes necesitan recibir ventilación mecánica, de tal manera que ésto ha estado ocurriendo desde el nacimiento de los cuidados intensivos hace 70 años" (Gattinoni, Chiumello, Caironi, Busana, Romitti, \& Brazzi, 2020).
Los escritores y editores concienzudos siempre han insistido en describir a los pacientes como si hubieran "recibido" ventilación mecánica en lugar de tener una "necesidad" de ventilación mecánica porque nunca se ha puesto de acuerdo completamente sobre quién la necesita. Lo que pudo haber parecido una sutileza sobre la semántica ahora tiene grandes repercusiones. Se está viendo en las publicaciones actuales sobre COVID-19 son diferentes tasas de ventilación mecánica invasiva en todo el mundo que siempre han existido, pero estas diferencias ahora son grandes porque se trata de un juego de interpretación de datos mundial de alto riesgo y una desesperación en aprender tanto como sea posible de las experiencias de los demás.

En una muestra de algunos de los estudios epidemiológicos más amplios de pacientes con COVID-19 hasta la fecha, "las tasas de ventilación mecánica invasiva entre los pacientes ingresados en las UCI oscilan entre el $29,1 \%$ en un estudio chino" (Wang, Lu, \& Chen, 2020) y "el 89,9\% en un estudio estadounidense y desde el $2,3 \%$ de los pacientes ingresados en el hospital hasta el 33,1\%" (Richardson, Hirsch, \& Narasimhan, 2020).

Muchos problemas, no relacionados con el virus en sí, están incluidos en estos informes epidemiológicos. Primero está la toma de decisiones clínicas, que siempre ha variado, incluso cuando se trata del síndrome de distrés respiratorio agudo "clásico".

Por ejemplo, en el estudio LungSafe (de pacientes sin COVID-19), a pesar de que todos cumplían los criterios de inclusión para el síndrome de dificultad respiratoria aguda, el $15 \%$ de los pacientes recibían ventilación no invasiva los primeros 2 días después de la inclusión en la cohorte (Bellani, Laffey, \& Pham, 2017).

En un estudio de variación realizado en pacientes que tenían lo que se denominó con-

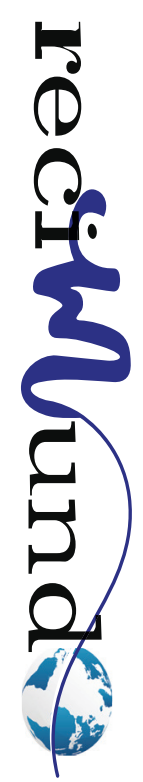


diciones de "evidencia sólida" para recibir ventilación no invasiva, los autores Mehta, Douglas, \& Walkey, (2017) encontraron; una gran variabilidad en el uso de esta modalidad en los hospitales de California, que van desde el 18,6\% de los pacientes en el nivel más bajo cuartil de hospitales hasta 42,0\% en el cuartil más alto, con variabilidad similar en la recepción de ventilación mecánica invasiva (p. 1667)

Nunca se ha podido ponernos de acuerdo sobre los desencadenantes del soporte ventilatorio, incluso con enfermedades mucho más conocidas y comprendidas que el COVID-19.

En segundo lugar, está la disponibilidad de recursos, que es una gran preocupación en este momento. La disponibilidad de recursos siempre ha variado entre países, lo que influye en los umbrales de ingreso a la UCI, la percepción de "necesidad" de ventilación mecánica y la duración de las terapias invasivas de soporte vital continuas. Sin embargo, lo que está relacionado con la variabilidad en los recursos, y es quizás la pieza más importante que recibe poco tiempo de transmisión, son las expectativas y preferencias de atención que a menudo se rigen por normas culturales.

Esta variabilidad en las preferencias está entrelazada con los recursos y siempre será un factor importante para comprender los datos que provienen de diferentes países; por ejemplo, Gray, Goodacre, Newby, \& Masson, (2008) publicaron un "gran ensayo controlado aleatorizado que compara la ventilación no invasiva con la oxigenoterapia convencional para pacientes con edema pulmonar cardiogénico agudo".

El ensayo fue "negativo" para su resultado primario; no hubo diferencia en la mortalidad a los 7 días entre los grupos. Pero si se mira más de cerca, se hace evidente que entre los que murieron dentro de los 7 días, un máximo del 30\% fueron colocados en un ventilador cuando su tratamiento comenzó a fallar (Gray, Goodacre, Newby, \& Masson, 2008)

Este estudio se realizó en el Reino Unido con aproximadamente siete veces menos camas de UCl que en los Estados Unidos. ¿Qué hubiera pasado si los pacientes hubieran recibido ventilación mecánica? ¿Es posible que se haya perdido una señal sin el uso de este soporte de rescate? No es posible saberlo. Y tampoco saber cuántas de esas decisiones de no colocar a los pacientes en ventiladores se debieron a la preferencia del paciente y la familia versus la disponibilidad de ventiladores en ese momento.

La transparencia con respecto a los recursos, las preferencias y la toma de decisiones clínicas puede ayudar al lector en la interpretación de los datos. Siempre que sea posible, debe incluirse información básica, como el país o la ubicación del estudio y la disponibilidad relativa de camas de la UCl. Por ejemplo, los patrones de atención cuando solo el $1 \%$ de las camas de un hospital son camas de UCI probablemente serán diferentes a los de un hospital donde constituyen el 20\% de las camas de los hospitales; proporcionar dicha información en los métodos de un estudio ayudará a los lectores a comprender los datos.

De manera similar, la información sobre las preferencias de atención, como cuántos pacientes prefirieron no recibir ventilación mecánica (debido a la edad, la comorbilidad u otra preferencia personal) ayudaría a interpretar los datos, como cuando se informa que solo el $20.2 \%$ de los que murieron con COVID -19 recibieron esta intervención (Xie, Tong, Guan, Du, \& Qiu, 2020).

Además, la transparencia con respecto a las prácticas clínicas, como el enfoque del uso de la ventilación mecánica invasiva, es importante. Como ejemplo, los autores Goyal, Choi, Pinheiro, Schenck, \& Chen, (2020) 
en su estudio "proporcionan la información de que utilizaron una estrategia de intubación temprana como explicación de su alta tasa $(33,1 \%)$ de ventilación mecánica entre los pacientes hospitalizados; incluso sería útil contar con más información sobre sus umbrales para la intubación". En otras palabras, cuanta más contextualización para el lector exista, mejor será.

\section{Mortalidad de los pacientes que reciben ventilación mecánica}

¿Cuál es la mortalidad de los pacientes que reciben ventilación mecánica con COVID-19? En algunos países, los titulares de los periódicos "afirmaron inicialmente que el $65 \%$ de los pacientes que reciben ventilación mecánica están muriendo, y estudios sobre los pacientes de Nueva York inicialmente incluía un resumen que indicaba que la mortalidad de los pacientes con ventilación mecánica era 88\%" (Richardson, Hirsch, \& Narasimhan, 2020).

Sin embargo, los denominadores que se utilizan son importantes. "En datos del Reino Unido y de Nueva York, los denominadores excluyeron a las personas que aún estaban en la UCl con ventilador" (Richardson, Hirsch, \& Narasimhan, 2020).

En un estudio de Italia, los que estaban en la UCl se incluyeron en el denominador, y desde entonces se corrigió el resumen de los datos de Richardson y sus colegas para informar el porcentaje de pacientes vivos, muertos y aún en la UCI para intentar evitar esta mala interpretación (Grasselli, Pesenti, \& Cecconi, 2020).

Es necesario, conocer el resultado para todos antes de poder sacar conclusiones firmes. La mortalidad puede ser más alta de lo que esperamos, pero excluir a todos aquellos que aún reciben atención está causando confusión a los lectores con menos comprensión de los principios epidemiológicos. Por lo tanto, asegurarse de que la incertidumbre se presenta en una forma que resalta claramente la gran cantidad de individuos que aún no tienen resultados claros o presentando la mortalidad estimada utilizando el rango de números posibles asumiendo los mejores y peores escenarios; en este momento, esos rangos parecen amplios debido a la gran cantidad de personas que aún reciben atención en las UCI. Lo que se puede decir a partir de estos datos es que parece que muchos pacientes que reciben ventilación mecánica pueden recibirla durante un período de tiempo prolongado, lo que requiere un seguimiento extenso para conocer sus resultados finales.

El objetivo de utilizar ventilación mecánica invasiva para pacientes con COVID-19 es universal y se requiere para salvar vidas a manera de reducir la mortalidad y asegurar que sea baja para todos, independientemente de la edad, comorbilidades o fragilidad, con una implementación juiciosa de la ventilación invasiva cuando se considere necesario. Sin embargo, algunos aspectos de la fisiología humana también son universales; la mortalidad de los pacientes colocados en ventiladores que tienen entre $80 \mathrm{y}$ 90 años o con comorbilidades graves siempre ha sido muy alta, incluso en el mejor de los momentos y en las mejores circunstancias. "En un estudio epidemiológico de 2010, el 50\% de las personas de 85 años o más que fueron ventiladas en los Estados Unidos murieron en el hospital" (Wunsch, Linde-Zwirble, \& Angus, 2010). Ciertos patrones comenzarán a replicarse en países y culturas que se harán más evidentes cuando finalmente se combinen todos estos datos para observar los promedios, eliminando los extremos. Probablemente habrá beneficios diferenciales de la ventilación mecánica entre los grupos de edad y aquellos con diferentes comorbilidades o fragilidad severa; es poco probable que podamos reducir la mortalidad a la misma tasa plana para todos con el uso de ventilación mecánica. 


\section{Conclusión}

La decisión de colocar a un paciente con COVID-19 en un ventilador no es clara y tampoco lo son los resultados. Nunca será completamente claro cómo o por qué estos datos de cada país se ven tan diferentes. Sin embargo, reconocer cuándo los patrones de atención y los resultados informados quedan fuera de las propias normas es esencial para hacer el mejor uso de estos datos para la atención en tiempo real.

Esto se debe probablemente a que los titulares que ensalzan una mortalidad muy elevada tienen consecuencias que van más allá del valor de la conmoción; y en tal sentido, los especialistas a cargo (médicos o enfermeras) pueden leerlos y sentir que lo que están haciendo es inútil más aun cuando la mayoría de los paciente por los cuales están arriesgando su vida están muriendo de forma masiva. Adicionalmente los familiares de los pacientes pueden entrar en pánico al ver el estado de empeoramiento de sus familiares y si sumado a ello se encuentran en países de menos recursos, se cuestionan si es necesario intentar adquirir ventiladores para este tipo de pacientes.

Sin embargo, no es tarea de la comunidad científica vigilar a la prensa. Pero es probable que los estudios epidemiológicos están siendo examinados por personas sin antecedentes científicos de una manera que rara vez ha ocurrido. Por lo tanto, es importante que se proporcionen datos lo más precisos posible, pero también presentados para limitar la capacidad de los lectores de captar un número específico que solo cuenta una parte de la historia. Puede ser más importante que antes pedirle a un "lector no científico" que mire un resumen o lea un manuscrito antes de que salga a la imprenta; y los editores que tienen más experiencia que la mayoría de los investigadores en la comunicación clara de datos deben ayudar a los escritores científicos a garantizar una presentación transparente de los resul- tados, dejando de lado cualquier enfoque en la generación de titulares o publicidad.

Entonces, mientras se examinan estos informes, se extrae lo que es universal y se pueda aplicar a la comprensión y atención de los pacientes a nivel local, se debe reconocer e informar sobre los enormes impulsores de las diferencias y estar atentos en la presentación de datos para minimizar la confusión en la interpretación. La variabilidad de los hallazgos siempre ha existido en los estudios de ventilación mecánica para pacientes críticamente enfermos. Por tanto, el nuevo COVID-19 no es una excepción, simplemente un amplificador de estas diferencias.

\section{Bibliografía}

World Health Organization. (2020, Jun 8). Coronavirus disease (COVID-19) pandemic. Retrieved from https://www.who.int/emergencies/ diseases/novel-coronavirus2019?gclid=CjCjOKCQjww_f2BRC-ARIsAP3zarGPN3jVnt36qWvYS5TZ 54HLi_8KsVwBpxNq4sEQsXCJ6_Vb7b_zsRoaArlBEALw_wcB

Bellani, G., Laffey, J., \& Pham, T. (2017). LUNG SAFE Investigators; ESICM Trials Group. Noninvasive ventilation of patients with acute respiratory distress syndrome: insights from the LUNG SAFE study. Am J Respir Crit Care Med, 67-77.

Berlin, D., Gulick, R., \& Martinez, F. (2020). Severe Covid-19. N Engl J Med, 1056.

Bolívar, J. (2015). Investigación Documental. México. Pax.

Castro, J. (2016). Técnicas Documentales. México. Limusa.

Davila, A. (2015). Diccionario de Términos Científicos. . Caracas: Editorial Oasis.

Gattinoni, L., Chiumello, D., Caironi, P., Busana, M., Romitti, F., \& Brazzi, L. (2020). COVID-19 pneumonia: different respiratory treatments for different phenotypes? . Intensive Care Med.

Goyal, P., Choi, J., Pinheiro, L., Schenck, E., \& Chen, R. (2020). Clinical characteristics of covid-19 in New York City. N Engl J Med.

Grasselli, G., Pesenti, A., \& Cecconi, M. (2020). Critical care utilization for the COVID-19 outbreak in Lombardy, Italy: early experience and forecast du- 
ring an emergency response. JAMA, 1545-1546.

Gray, A., Goodacre, S., Newby, D., \& Masson, M. (2008). Noninvasive ventilation in acute cardiogenic pulmonary edema. N Engl J Med, 142-151.

Mehta, A., Douglas, I., \& Walkey, A. (2017). Evidence-based utilization of noninvasive ventilation and patient outcomes. Ann Am Thorac Soc, 16671673.

Richardson, S., Hirsch, J., \& Narasimhan, M. (2020). Presenting characteristics, comorbidities, and outcomes among 5700 patients hospitalized with COVID-19 in the New York city area. JAMA, 20522059.
Slutsky, A. (2015). History of Mechanical Ventilation. From Vesalius to Ventilator-induced Lung Injury. Am J Respir Crit Care Med, 1106-1115.

Wang, Y., Lu, X., \& Chen, H. (2020). Clinical course and outcomes of 344 intensive care patients with COVID-19. Am J Respir Crit Care Med.

Wunsch, H., Linde-Zwirble, W., \& Angus, D. (2010). The epidemiology of mechanical ventilation use in the United States. Crit Care Med, 1947-1953.

Xie, J., Tong, Z., Guan, X., Du, B., \& Qiu, H. (2020). Clinical characteristics of patients who died of coronavirus disease 2019 in China. JAMA Netw Open.

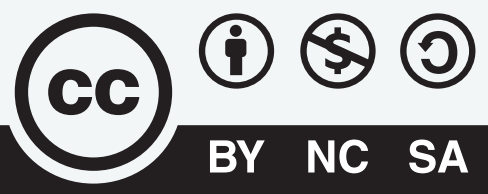

CREATIVE COMMONS RECONOCIMIENTO-NOCOMERCIAL-COMPARTIRIGUAL 4.0.

\section{CITAR ESTE ARTICULO:}

Párraga Gusqui, G. G., Arias Muñoz, C. A., Guamán Guaranga, C. P., \& Rivera Moreira, E. A. (2021). Ventilación mecánica en pacientes con COVID-19. RECIMUNDO, 5(2), 203-211. https://doi.org/10.26820/recimundo/5.(2). abril.2021.203-211 